\title{
Current Status of Automatic Serial Sections for 3D Electron Microscopy
}

\author{
Hyosun Choi ${ }^{1, \dagger}$, Min Kyo Jung ${ }^{2, \dagger}$, Ji Young Mun ${ }^{1,3 *}$ \\ ${ }^{1}$ BK21 Plus Program, Department of Senior Healthcare, Graduate School, Eulji University, Daejeon 34824, Korea \\ ${ }^{2}$ Department of Convergence Medicine, Asan Institute for Life Sciences, Asan Medical Center, \\ University of Ulsan College of Medicine, Seoul 05505, Korea \\ ${ }^{3}$ Department of Biomedical Laboratory Science, College of Health Science, Eulji University, Seongnam 13135, Korea
}

\author{
${ }^{\dagger}$ First authors were equally \\ contributed. \\ *Correspondence to: \\ Mun JY, \\ Tel: +82-31-740-7380 \\ Fax: +82-31-740-7354 \\ E-mail: mjy1026@gmail.com
}

Received March 20, 2017

Revised March 30, 2017

Accepted March 30, 2017
The automatic equipment for three-dimensional electron microscopy (3DEM) can acquire serial sections of a large sample in a relatively short time, and is especially suitable for the connectomics, which is a field related to understanding the brain structure as a whole. As many results obtained through 3DEM using automatic serial sections have been published in the field of brain research, many researchers continue to apply this technique to various samples. We reviewed the equipment for automatic serial sectioning, the block preparation method, the limitations of 3DEM, and future directions.

Key Words: Automatic serial sections, Three-dimensional electron microscopy, Correlative light and volume electron microscopy

\section{INTRODUCTION}

Researchers at world-class imaging centers use automatic serial sectioning techniques for three-dimensional (3D) reconstruction of tissues. In 2015, Jeff William's group at Harvard University reconstructed the 3D structure of the $1,500 \mu^{3}$ neocortex area using automated serial sections to tape (ATUM) at nanometer resolution (Kasthuri et al., 2015). In addition, serial sectioning techniques using serial blockface (SBF) imaging (3VEIW, volume scope [VS], and focused ion beam scanning electron microscopy, FIB-SEM) are established in many research institutes (Denk \& Horstmann, 2004; Kim et al., 2016; Miyazaki et al., 2014). Researchers are now focusing on improving the techniques for rapid imaging of large areas. According to these global trends, the use of automatic serial sectioning techniques for $3 \mathrm{D}$ electron microscopy (3DEM) is expected to increase. Therefore, there is a need for appropriate sampling methods to be applied for various samples. However, owing to the lack of research in this field, sufficient protocols are not available for various samples (block preparation, staining, and imaging methods for each sample). Therefore, it is time to review the current techniques and determine the needs for the future.

\section{STATUS}

At present, automatic serial sectioning techniques are classified into SBF imaging and ATUM (Fig. 1). The scanning electron microscope equipped with a diamond knife (3VIEW, VS) or a FIB can produce a series of block-face images (SBFSEM) continuously for automatic serial sections for 3DEM (Cabezon et al., 2017; Miyazaki et al., 2014). In addition, an ATUM was developed at the Massachusetts Institute of Technology (MIT) for connectomics. This ATUM is a system for automatically moving serial sections to a tape through a conveyor belt (Kasthuri et al., 2015). Each serial section collected on the tape has the advantage of specific staining, including immunostaining (Jung et al., 2016). With the global trend to boost brain research, the Korean Brain Research Institute (KBRI) also announced a five-year plan for the "High

(a) This is an open-access article distributed under the terms of the Creative Commons Attribution Non-Commercial License (http://creativecommons.org/licenses/by-nc/4.0) which permits unrestricted noncommercial use, distribution, and reproduction in any medium, provided the original work is properly cited.

Copyrights @ 2017 by Korean Society of Microscopy 


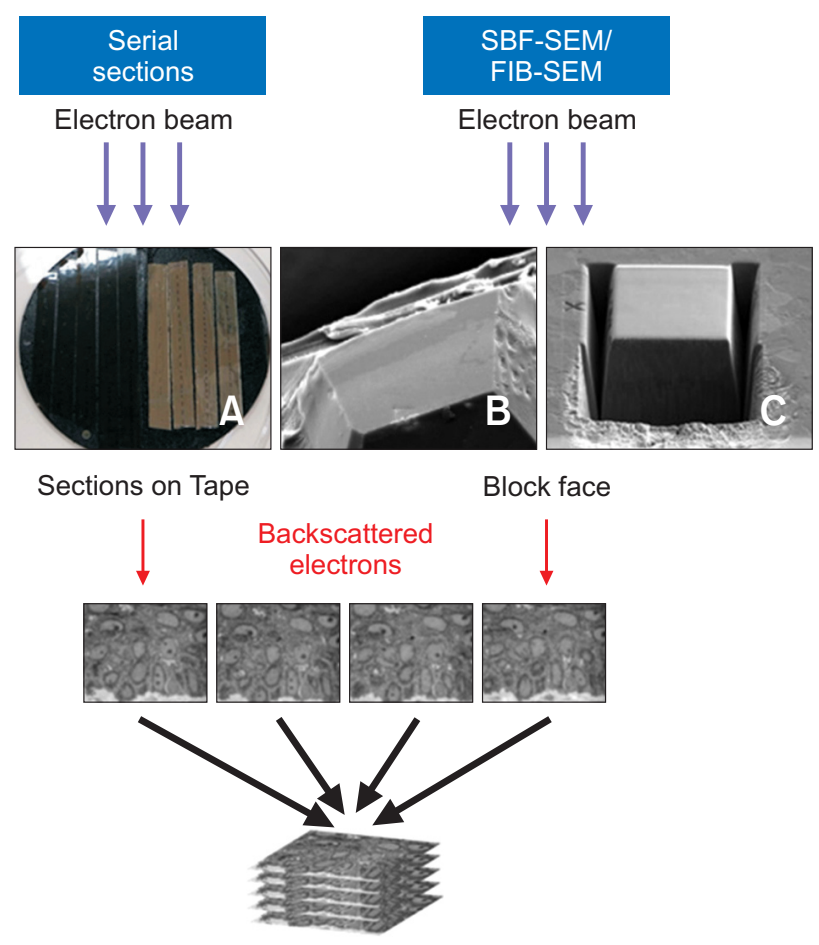

Fig. 1. Types of automatic serial sections for three-dimensional electron microscopy. (A) Automated serial sections to tape. The sections are picked up from the water surface onto the tape which moves like a conveyor belt. A wafer with sections on tapes for imaging in an scanning electron microscopy (SEM). (B) 3VIEW or volume scope (VS, TENEO) Microtome is attached to the inner side of the SEM. (C) focused ion beam scanning (FIB)-SEM system. The gallium ion gun is mounted on the SEM vacuum chamber and the focused ion beam moves across the sample surface for milling. SBF, serial block-face. resolution brain map" in 2016. Currently, connectome is used as a term for the network of the brain, but all cell connections can be included in a connectome in a broad sense of the term. In October 2016, the Broad Institute at MIT, Harvard University, Cambridge Sanger Centre, and Wellcome Trust Foundation launched a human cell mapping project involving about 35 trillion cell functions and characteristics that make up the human body (source of data base [https://www. humancellatlas.org/]). To study the structural changes in cells, our group prefers to use $3 \mathrm{D}$ structure analysis employing serial sections. For example, we studied the melanocyte in the human skin epidermis through $3 \mathrm{D}$ reconstruction.

Laser treatment in clinical research is effective for hyperpigmentation when melanin is overproduced and pigmentation deposits on the skin. However, research on the mode of action for the treatment is lacking. In this study, we observed the changes in the $3 \mathrm{D}$ structure of melanocytes after laser treatment of the skin with hyperpigmentation. The twodimensional (2D) images obtained showed that melanin, the pigment that imparts color to the human skin, decreased clearly in the melanocytes after laser treatment. For the 3D structure, elanocytes were serially imaged with a 3VIEW SBF-SEM system, and a 3D structure was reconstructed. We showed that the laser treatment induces changes in the structure of the melanocytes and causes a decrease in the dendrites, suggesting problems in melanin transfer. (Mun et al., 2011). Since the melanocytes are 5 6 times longer than their cell body themselves and spread out over a large area, the 3VIEW serial sectioning technique was necessary to produce

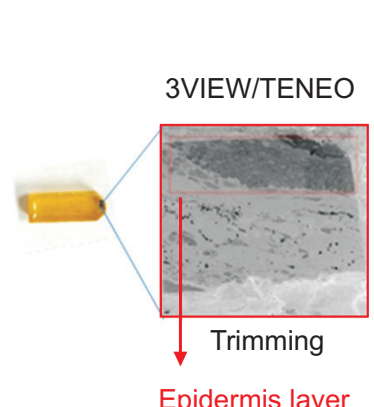

of skin

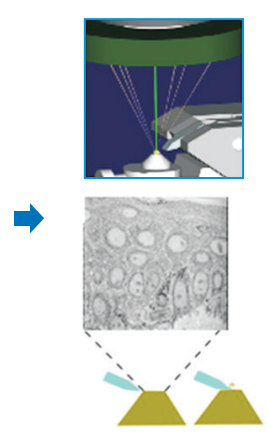

Serial sections
Diamond knife in SEM

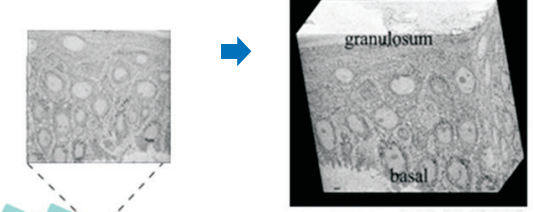

3D volume

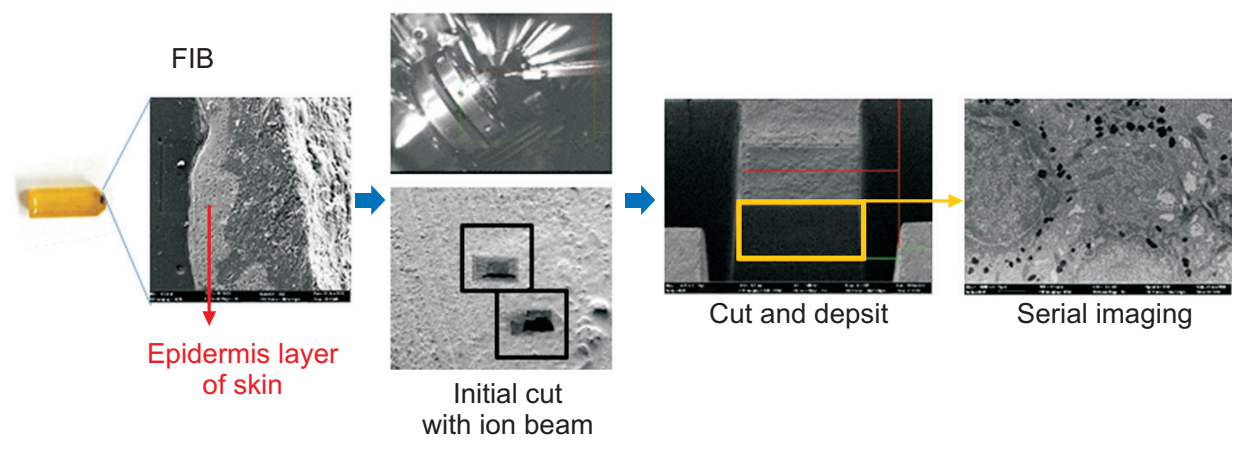

Fig. 2. Serial images of epidermis layer in skin. Serial images using 3VIEW or volume scope (VS, TENEO) system. Serial images showed whole epidermis layer of human skin. Its resolution was enough to study location of melanin granule. Serial images using focused ion beam (FIB). The field was narrower than 3VIEW or VS (TENEO), but the resolution was enough to investigate interactions of neighbor cells. SEM, scanning electron microscopy. 
images of the large number of melanocytes and keratinocyte layers in an area of $50 \times 50 \mu \mathrm{m}^{2}$ (4 5 layers of cells). This technique was effective for investigating the structural changes in the melanocytes before and after laser treatment. We also tried to image the melanocytes and keratinocytes through FIB-SEM and VS systems to study the melanin transfer (Fig. 2). The VS provided serial images of multiple cell layers over a wide area, similar to the 3VIEW. However, the FIB-SEM had a limitation that it could obtain images only in a narrow region as compared to the range of 3VIEW or VS. Thus, both SBFSEM techniques are suitable for producing serial images with sufficient resolution to determine the shape of the melanocyte and keratinocyte and the position of the melanosome (Mun et al., 2017).

Although SBF-SEM microscopy has advantages such as a relatively short time for sectioning as compared to the conventional transmission electron microscopy (TEM) with ultramicrotomy, there are some limitations. For example, since SBF-SEM requires higher contrast and electronic conductivity as compared to TEM imaging, the equipment has to be designed to fix the tissue and then stain it with uranyl acetate, lead aspartate, and osmium solution. Further, image quality is not consistent because of the charging effect due to excessive exposure to the electron beam despite staining the tissue with various chemicals. Therefore, EM researchers worldwide have continuously worked to improve and develop new staining techniques to overcome the limitations. Tapia and co-workers used copper sulfate for the SBF-SEM block to realize less damage by electronic charge. They reconstructed the 3D structure of synaptic vesicles at sufficiently high resolution for observing the connection between synapses; their results were reported in Nature Protocol (Tapia et al., 2012). In the Max-Planck Institute, the balance of attachment and diffusion between the staining solution and specimens was optimized to improve permeation, and this technique was used for staining mouse neocortex to improve the previous tissue-staining techniques. The stained tissues were used for serial sections, and a 3D structure was reported in Nature Communications (Hua et al., 2015). It was found that periodic acid-thiocarbohydrazide-OsO $\mathrm{O}_{4}$ (PATCO) has high permeability to tissues, but it seems to be especially good for staining the myelinated neurites. On the other hand, brainwide reduced-osmium staining with pyrogallol-mediated amplification (BROPA) was suitable for staining large areas of the brain tissue. In 2016, Nobuhiko Ohno's group in Japan improved the quality of images by adding black carbon during resin preparation to eliminate the electron charging effect of samples during automatic serial sectioning for 3D volume imaging, and reported the results in Scientific Reports (Nguyen et al., 2016). Another limitation of the SBFSEM is that the immunogold technique for labeling specific cells in the conventional TEM cannot be used in SBF-SEM microscopy. Researchers at the MIT developed Enhanced APX (Ascorbate Peroxidase) that can be applied to whole cells as an alternative to horseradish peroxidase (HRP), which can be used only in specific areas of the cell. The group developed a new APEX tag ("engineered APX") with the target protein gene and 3,3'-diaminobenzidine (DAB) oxidation to make it visible by EM (Martell et al., 2012). At the Heidelberg Institute, the 3D structure of specific presynaptic terminals was reconstructed by labeling the neurons via the fusion of high-density DAB with GFP-tagging; the results were published in PLOS One (Horstmann et al., 2013). Researchers at the Harvard University used viral vectors to utilize electrondense peroxidases for staining specific neurons. They used this technique with 3DEM through ATUM (Joesch et al., 2016). In Australia, Filip Braet's group qualitatively and quantitatively reported the advantages and disadvantages of the five current SBF-SEM sample preparation protocol for liver tissue in 2016 and produced blocks using selected methods for correlative light and electron microscopy (CLEM), combining volume images with confocal laser scanning microscopy (CLSM) and EM. They reconstructed 3D structures using serial sections obtained by CLEM, and reported the results in Scientific Reports (Shami et al., 2016). In particular, correlative light and volume electron microscopy (CLVEM) were attempted as new research techniques that combine the results of CLSM and 3DEM with serial images (Lucas et al., 2012).

\section{FUTURE DIRECTIONS}

Correlative microscopy aims to utilize the advantages of both the light microscope and electron microscope simultaneously. This is the latest EM technique to investigate the location of the target protein easily by light microscopy and then study the ultrastructure by block preparation, sectioning, staining, and EM at high resolution. In addition, the cells should be marked using a specific glass and holder system to observe the same cells via both light microscopy and EM. For testing the technique, our group observed the location of a specific protein in cells through fluorescence microscopy, and then obtained EM images of the same region of the cells using a marked EM grid or a coverslip. In this study, we studied the location of nanoparticles in the cell through CLEM and reconstructed the 3D structure (unpublished data; Jung et al.). Further, the 3D structure of specific brain cells labeled by the array tomography technique can be reconstructed using serial sections with ATUM, immunostaining, and CLEM (Collman et al., 2015). Since the serial sections obtained by ATUM or array tomography (Fig. 3) can be stained after sectioning, the immunostaining technique for the general electron microscope can be utilized for sections on tapes or coverslips. The intensity and selectivity of the staining can be controlled by the fixative solution used; the characteristics, concentration, 


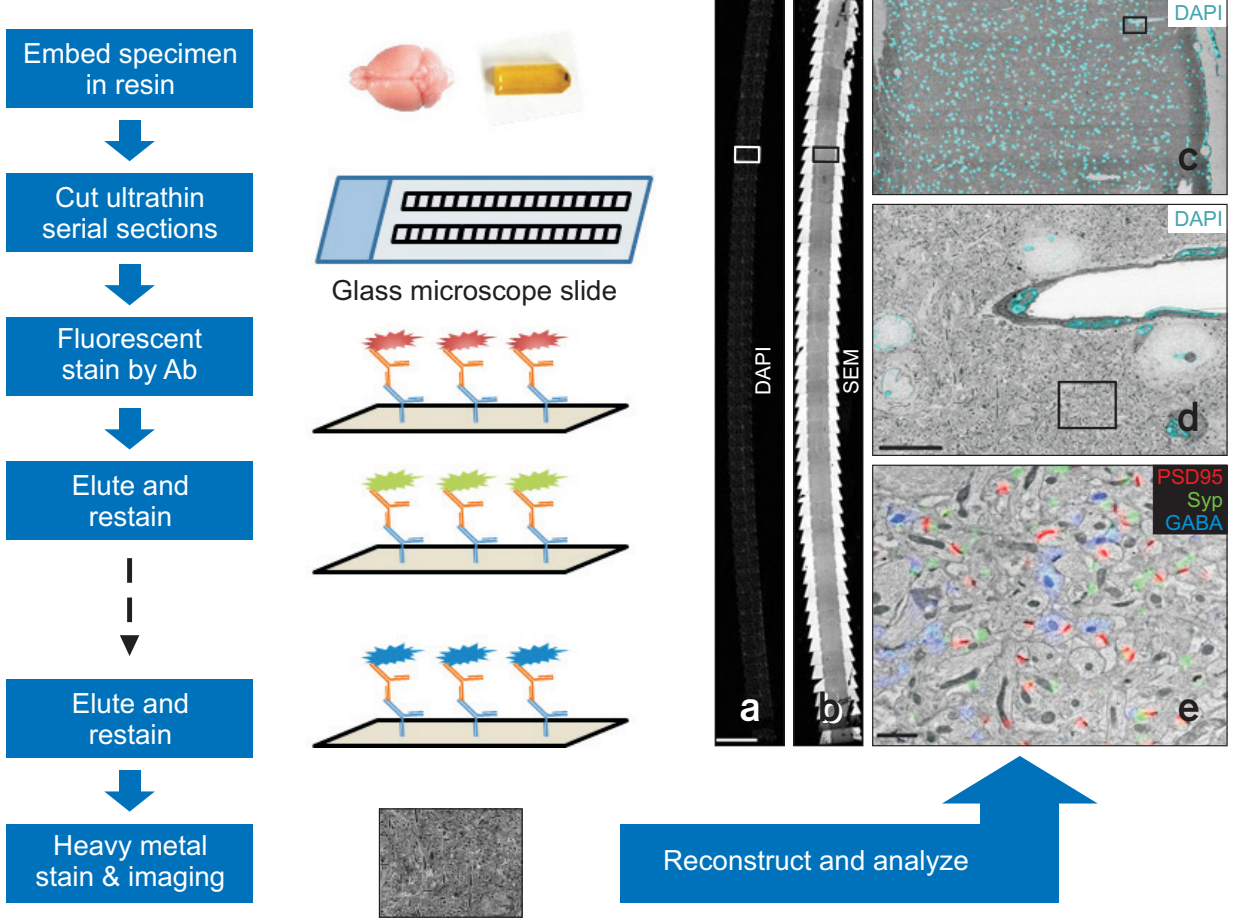

Fig. 3. Schematic representation of the array tomography. A tissue specimen is embedded in a resin and cut into ribbons of serial ultrathin $(50 \sim 200 \mathrm{~nm})$ sections. The array is labeled with fluorescent antibodies or other fluorescent stains and imaged, then can be repeatedly eluted, restained. Finally it can be stained with heavy metals and imaged under a scanning electron microscope. In right (a e), the example for array tomography was reproduced from Collman et al. (2015) (J. Neurosci. 35, 5792-5807) with permission from the publisher.
$\mathrm{pH}$, and the ions of the staining solution; the time and temperature of the staining; and the thickness of the sections. Currently, 3VIEW (in KBRI and Dankook University), VS (Korea Institute of Science and Technology), and FIBSEM (Seoul National University National Instrumentation Center for Environmental Management (NICEM) and Gumi Electronics \& Information Technology Research Institute) are available for obtaining automatic serial sections for 3DEM in Korea. Several research groups have published results based on images obtained using each device, but most of the researchers employed previously reported block staining techniques. Therefore, it is clear that a leading group for 3DEM is necessary to verify and improve the current techniques to apply and utilize the techniques for various samples.

Fixation is largely based on the chemical method applied for 3DEM, but the chemical fixative can lead to ultrastructural changes and decrease in the reactivity to antibody (Bittermann et al., 1992; Mun et al., 2008). Cryofixation, on the other hand, is an immobilization method employed at low temperatures during a short time that is optimized for the location of proteins by minimizing the aldehyde content, which prevents antigen-antibody reactions (Mun et al., 2008). Samples can be fixed using the high-pressure freezing method under a high pressure of 2100 bar or more, and cryoprotectants, including hexadecene or bovine serum albumin, can help reduce the damage caused by ice crystals. In the system, osmification and block staining using heavy metals can be conducted in acetone solution to increase the electron density of the frozen sample, and the resin block can be used for the SBF-SEM techniques. The tissue obtained after low-temperature polymerization without block staining can be observed via serial images through ATUM after protein labeling and double staining with uranium acetate and lead citrate.

Staining depends on electrostatic binding; hence, buffers containing ions in addition to the staining solution play an important role. In addition, an alcoholic staining solution can improve the contrast. Therefore, it is important to establish appropriate and even staining conditions by modifying various conditions such as ion concentration, $\mathrm{pH}$, temperature, incubation time, primary oxidant, heavy metal concentration, polar protic solvents, polar aprotic solvents, detergent, and chelator.

\section{CONCLUSIONS}

To summarize, the development of the resin and staining method for reducing damage by electron charging will help obtain serial images without the charging effect and will overcome the limitation of 3DEM through SBFSEM. Correlative volume electron microscopy, which is a combination of immuno-EM and correlative microscopy and automatic serial sections for 3DEM to target specific proteins, can be used for the $3 \mathrm{D}$ reconstruction of specific cells. In a variety of biological studies including brain research, 3D correlative microscopy (CLVEM) can be applied to various studies. 


\section{CONFLICT OF INTEREST}

No potential conflict of interest relevant to this article was reported.

\section{ACKNOWLEDGMENTS}

This research was supported by the Basic Science Research Program through the National Research Foundation of Korea (NRF) funded by the Ministry of Science, ICT \& Future Planning (2015R1C1A1A02037153), and Global Frontier Research Grant, NRF-2015M3A6A4065729.

\section{REFERENCES}

Bittermann A G, Knoll G, Nemeth A, and Plattner H (1992) Quantitative immuno-gold labelling and ultrastructural preservation after cryofixation (combined with different freeze-substitution and embedding protocols) and after chemical fixation and cryosectioning. Analysis of the secretory organelle matrix of Paramecium trichocysts. Histochemistry 97, 421-429.

Cabezon I, Auge E, Bosch M, Beckett A J, Prior I A, Pelegri C, and Vilaplana $J$ (2017) Serial block-face scanning electron microscopy applied to study the trafficking of 8D3-coated gold nanoparticles at the bloodbrain barrier. Histochem. Cell Biol. doi: 10.1007/s00418-017-1553-9.

Collman F, Buchanan J, Phend K D, Micheva K D, Weinberg R J, and Smith S J (2015) Mapping synapses by conjugate light-electron array tomography. J. Neurosci. 35, 5792-5807.

Denk W and Horstmann H (2004) Serial block-face scanning electron microscopy to reconstruct three-dimensional tissue nanostructure. PLoS Biol 2, e329.

Horstmann H, Vasileva M, and Kuner T (2013) Photooxidation-guided ultrastructural identification and analysis of cells in neuronal tissue labeled with green fluorescent protein. PLoS One 8, e64764.

Hua Y, Laserstein P, and Helmstaedter M (2015) Large-volume en-bloc staining for electron microscopy-based connectomics. Nat. Commun. 6, 7923.

Joesch M, Mankus D, Yamagata M, Shahbazi A, Schalek R, Suissa-Peleg A, Meister M, Lichtman J W, Scheirer W J, and Sanes J R (2016) Reconstruction of genetically identified neurons imaged by serialsection electron microscopy. Elife 5, e15015.

Jung M K, Kang M S, and Mun J Y (2016) Techniques and applications of three-dimensional electron microscopy in entomological research. Entomol. Res. 46, 374-380.

Kasthuri N, Hayworth K J, Berger D R, Schalek R L, Conchello J A, Knowles-Barley S, Lee D, Vazquez-Reina A, Kaynig V, Jones T R, Roberts M, Morgan J L, Tapia J C, Seung H S, Roncal W G, Vogelstein J T, Burns R, Sussman D L, Priebe C E, Pfister H, and Lichtman J W (2015) Saturated reconstruction of a volume of neocortex. Cell $\mathbf{1 6 2}$ 648-661.
Kim G H, Lee S H, and Lee K J (2016) Reconstruction of neural circuits using serial block-face scanning electron microscopy. Appl. Microsc. 46, 100-104.

Lucas M S, Gunthert M, Gasser P, Lucas F, and Wepf R (2012) Bridging microscopes: 3D correlative light and scanning electron microscopy of complex biological structures. Methods Cell Biol. 111, 325-356.

Martell J D, Deerinck T J, Sancak Y, Poulos T L, Mootha V K, Sosinsky G E, Ellisman M H, and Ting A Y (2012) Engineered ascorbate peroxidase as a genetically encoded reporter for electron microscopy. Nat. Biotechnol. 30, 1143-1148.

Miyazaki N, Esaki M, Ogura T, and Murata K (2014) Serial block-face scanning electron microscopy for three-dimensional analysis of morphological changes in mitochondria regulated by $\mathrm{Cdc} 48 \mathrm{p} / \mathrm{p} 97$ ATPase. J Struct. Biol. 187, 187-193.

Mun J Y, Jeong S Y, Kim J H, Han S S, and Kim I H (2011) A low fluence Q-switched Nd:YAG laser modifies the 3D structure of melanocyte and ultrastructure of melanosome by subcellular-selective photothermolysis. J. Electron. Microsc. (Tokyo) 60, 11-18.

Mun J Y, Kim I H, and Choi H S (2017) Three-dimensional image analysis of melanocyte in human skin. Biophys. J. 112, 578a.

Mun J Y, Lee K E, and Han S S (2008) Techniques for cryo-elelctron tomography in biological field. Korean J. Microsc. 38, 73-79.

Nguyen H B, Thai T Q, Saitoh S, Wu B, Saitoh Y, Shimo S, Fujitani H, Otobe $\mathrm{H}$, and Ohno N (2016) Conductive resins improve charging and resolution of acquired images in electron microscopic volume imaging. Sci. Rep. 6, 23721.

Shami G J, Cheng D, Huynh M, Vreuls C, Wisse E, and Braet F (2016) 3-D EM exploration of the hepatic microarchitecture-lessons learned from large-volume in situ serial sectioning. Sci. Rep. 6, 36744.

Tapia J C, Kasthuri N, Hayworth K J, Schalek R, Lichtman J W, Smith S J, and Buchanan J (2012) High-contrast en bloc staining of neuronal tissue for field emission scanning electron microscopy. Nat. Protoc. 7, 193-206. 